\title{
Detection of cartilage matrix degradation by autofluorescence lifetime
}

Hugh B Manning ${ }^{1,2,3}$, Mohammad B Nickdel ${ }^{3}$, Kazuhiro Yamamoto ${ }^{3}$, João L Lagarto $^{2}$, Douglas J. Kelly ${ }^{2}$, Clifford B Talbot ${ }^{2}$, Gordon Kennedy ${ }^{2}$, Jayesh Dudhia ${ }^{4}$, John Lever ${ }^{5}$, Christopher Dunsby ${ }^{2}$, Paul French ${ }^{2 *}$ and Yoshifumi Itoh ${ }^{3 *}$

${ }^{1}$ Chemical Biology Centre, Department of Chemistry, Imperial College London, London, SW7 2AZ, UK

${ }^{2}$ Department of Physics, Imperial College London, London, SW7 2AZ, UK

${ }^{3}$ Kennedy Institute of Rheumatology, University of Oxford, London W6 8LH, UK

${ }^{4}$ Royal Veterinary College, Department of Veterinary Clinical Sciences, Hawkshead Lane, Hatfield, Herts, AL9 7TA, UK

${ }^{5}$ Department of Bioengineering, Imperial College London, SW7 2AZ, UK

Running Title: Autofluorescence lifetime detects cartilage degradation

\author{
*All correspondence should be addressed to: \\ Yoshifumi Itoh, $\mathrm{PhD}$ \\ Kennedy Institute of Rheumatology \\ University of Oxford \\ 65 Aspenlea Road \\ London W6 8LH \\ Tel: +44 (0)20 83834410 \\ Fax: +44 (0)20 83834760 \\ E-mail: yoshi.itoh@kennedy.ox.ac.uk \\ Paul French, PhD \\ Photonics Group \\ Physics Department \\ Imperial College London \\ South Kensington Campus \\ London SW7 2AZ, UK \\ Tel: $\quad+44-(0) 20-75947706$ \\ Fax : +44-(0)20-7594 7714 \\ Email: paul.french@imperial.ac.uk
}




\begin{abstract}
Cartilage is a vital organ to maintain joint function. Upon arthritis, proteolytic enzymes initiate degradation of cartilage extracellular matrix (ECM) resulting in eventual loss of joint function. However, there are only limited ways of noninvasively monitoring early chemical changes in cartilage matrix. Here we report that the autofluorescence decay profiles of cartilage tissue are significantly affected by proteolytic degradation of cartilage ECM and can be characterised by measurements of the autofluorescence lifetime (AFL). A compact multidimensional fluorometer coupled to a fibre-optic probe was developed for single point measurements of AFL and applied to cartilage that was treated with different proteinases. Upon treating cartilage with bacterial collagenase, trypsin or matrix metalloproteinase 1 , a significant dose and time dependent decrease of AFL was observed. Our data suggest that AFL of cartilage tissue is a potential non-invasive readout to monitor cartilage matrix integrity that may contribute to future diagnosis of cartilage defects as well as monitoring the efficacy of anti-joint therapeutic agents.
\end{abstract}

\title{
Keywords
}

Cartilage, collagen, aggrecan, auto fluorescent lifetime

\section{Abbreviations}

AFL: autofluorescence lifetime

FL: fluorescence lifetime

MMP: matrix metalloproteinase

OA: osteoarthritis

RA: rheumatoid arthritis 


\section{INTRODUCTION}

Cartilage is a unique avascular tissue composed of a dispersed population of chondrocytes and rich extracellular matrix (ECM). Cartilage ECM is predominantly composed of type II collagen and aggrecan proteoglycans (about $45 \%$ each) with other minor components including small leucine rich proteoglycans and type IX and XI collagens (Heinegård et al., 2001; Sledge et al., 2001). In arthritic cartilage, these components are degraded by proteolytic enzymes, and the main proteinases responsible for cartilage matrix degradation are matrix metalloporeinases (MMPs) and disintegrin-metalloporteinases with thrombospondin motif (ADAMTSs)(Murphy and Nagase, 2008). Collagen is degraded by collagenases belonging to the MMPs family and aggrecan by MMPs and ADAMTSs. It is proposed that aggrecan is degraded in the early stage of arthritis and is followed by collagen degradation. This earlier aggrecan depletion is thought to be due to the action of aggrecanases belonging to ADAMTSs family including ADAMTS-4 and -5 (Murphy and Nagase, 2008; Stanton et al., 2011). Indeed, cartilage matrix of ADAMTS-5 null mice was shown to be largely protected from challenges against antigen-induced arthritis (Stanton et al., 2005) and a mouse osteoarthritis (OA) model has been induced by surgical destabilisation of knee joints (Glasson et al., 2005). In human OA cartilage, cleaved collagen products have been detected in cartilage tissue from the articular surface to deeper areas where extensive degeneration is apparent (Hollander et al., 1995; Wu et al., 2002). Together, these proteolytic modifications of cartilage ECM by multiple proteinases result in loss of joint function, which is largely irreversible. Therefore there is a need to detect early biochemical changes of cartilage matrix, but the currently used methods of x-ray and magnetic resonance imaging detect only structural alteration of joint lesions. 
Measurement of autofluorescence lifetime (AFL) has been shown to detect qualitative chemical changes of tissue (Elson et al., 2004; Manning et al., 2008b; Talbot et al., 2010). Fluorescence is the spontaneous emission of a photon as an electron relaxes to its ground state following excitation to a higher energy level. The average time during which an excited electron remains in the excited state is defined as the fluorescence lifetime (FL) and this intrinsic parameter can distinguish different molecular species. FL measurements are independent of fluorophore concentration and the efficiency of excitation and detection of the emission, making them more robust than fluorescence intensity measurements. For some fluorophores, the FL can be modified by changes in the local chemical or physical environment and so can be utilised to report on variations in, e.g. viscosity, $\mathrm{pH}$, refractive index, solvent polarity and ionic concentrations (Lakowicz, 2006). One of the major fluorophores present in the cartilage matrix is collagen (Georgakoudi et al., 2002; Menter, 2006). Because interstitial collagen polypeptides contain only 7 tryptophans, 10 tyrosines and 25 phenylalanines out of about 1,000 amino acids, the major source of fluorescent signal in collagen matrix is its intermolecular cross-links (Wagnieres et al., 1998). In intact cartilage, collagens exist as fibriler forms that are surrounded by aggrecan and other minor ECM components, including small leucine-rich proteoglycans, and the collagen AFL is thought to be sensitive to alterations in these local components. In this study we tested this hypothesis by studying cartilage tissue treated with various proteinases as a starting point and have demonstrated that AFL can detect the degradation of ECM.

\section{RESULTS}

Autofluorescence lifetime of normal cartilage 
A major cartilage component, type II collagen, is considered to be the main source of autofluorescence in the tissue. Although it contains limited numbers of aromatic amino acids, including 7 tryptophans, 10 tyrosines and 25 phenylalanines, the fluorescence of these molecules is excited only in the far UV (Lakowicz, 2006). For excitation in the more accessible near UV spectral region at $355 \mathrm{~nm}$, we expect to observe fluorescence from the cross-links between collagen molecules (Wagnieres et al., 1998). As shown in Figure 1, our measured excitation-emission matrix of bovine type II collagen indicates that excitation at $355 \mathrm{~nm}$ produces peak autofluorescence emission $390-430 \mathrm{~nm}$, which is readily detected.

To investigate potential autofluorescence lifetime contrast, we analyzed porcine articular cartilage using our multidimensional fluorometer and recorded the fluorescence emission resolved with respect to time and wavelength. Figure 2A shows the spectro-temporal characteristics of the cartilage autofluorescence excited with picosecond pulses at $355 \mathrm{~nm}$ and figure $2 \mathrm{~B}$ shows the time-integrated emission spectrum. The temporal decay profile of the cartilage autofluorescence was fitted to both a single exponential and a double exponential decay profile and the double exponential decay model, $I(t)=\alpha_{1} e^{-t / \tau_{1}}+\alpha_{2} e^{-t / \tau_{2}}$ was found to provide a superior fit with smaller weighted residuals and a $\chi^{2}$ close to unity. The values obtained for the corresponding fit parameters are given in Table 1. Our data indicates an autofluorescence decay profile of this cartilage tissue comprising a $12.82 \mathrm{~ns}$ AFL component (providing $76.4 \%$ of the total fluorescence signal) and a $1.74 \mathrm{~ns}$ AFL component $(23.6 \%)$, resulting in a mean AFL of 10.2 ns. The mean lifetime was not observed to vary with emission wavelength. Figure $2 \mathrm{C}$ shows a typical autofluorescence decay profile recorded at $415 \mathrm{~nm}$, together with the temporal profile of the instrument response function (IRF) and the fit to the double exponential decay 
model. Figure 2D shows the results of repeated measurements of porcine articular cartilage $(n=21)$ provided a mean AFL of $9.9 \pm 0.2 \mathrm{~ns}$ (mean \pm SEM) (Figure $2 \mathrm{D})$. The AFL was found to vary between different areas of cartilage and different cartilage pieces, potentially reflecting differences of chemical environment of collagen fibrils in each area.

\section{Fluorescence changes associated with cartilage degradation}

To examine effects of proteolytic degradation of cartilage on AFL, frozen and thawed porcine articular cartilage was treated with bacterial collagenase. A high concentration of collagenase $(1 \mathrm{mg} / \mathrm{ml})$ dissolved cartilage tissues almost completely within $18 \mathrm{~h}$ at $37^{\circ} \mathrm{C}$ (data not shown), indicating that this enzyme can degrade both of the major components of cartilage, i.e. collagen and aggrecan. Thus the action of bacterial collagenase on cartilage can simulate the condition of arthritic cartilage being degraded by multiple proteinases. We treated porcine cartilage with different concentrations of the collagenase ranging from $10 \mu \mathrm{g} / \mathrm{ml}$ to $100 \mu \mathrm{g} / \mathrm{ml}$ at $37^{\circ} \mathrm{C}$ for $16 \mathrm{~h}$. Treating cartilage with $100 \mu \mathrm{g} / \mathrm{ml}$ for $16 \mathrm{~h}$ resulted in $50 \%$ of collagen release from cartilage pieces (data not shown). The autofluorescence of each cartilage sample was measured five times using an optical fiber probe attached to the multidimensional fluorometer with picosecond pulsed excitation at $355 \mathrm{~nm}$ and emission measured at $415 \mathrm{~nm}$. Figure 3A shows the autofluorescence decay traces and Figure 3B shows the variation of calculated mean AFL for a range of collagenase concentrations. The data indicate that enzyme treatment decreases the mean AFL of cartilage and there is a clear inverse correlation between mean AFL and concentration of collagenase treated as demonstrated by a good fit to a linear equation $\left(\mathrm{r}^{2}=0.90\right)$. The behavior of the mean AFL lifetime can be traced back to both a decrease in the lifetime of the long component of the double exponential fit (data not shown) and an increased relative 
contribution from the shorter component (Figure 3C). Interestingly, the collagenase treatment did not affect the autofluorescence emission spectrum of cartilage (Figure 3D), suggesting that the relative concentrations of constituent fluorophores was not affected by the enzyme treatment.

The above experiments were carried out by measuring a number of cartilage pieces treated with the enzyme. To confirm that the shift of AFL truly reflects changes caused by the enzyme treatment and was not due to heterogeneity of cartilage tissues, we monitored a three cartilage samples over a period of treatment with the enzyme and a further three samples that were untreated. The same area of the same cartilage piece were analyzed at each time point of incubation. As shown in Figure 4, the AFL of control cartilage was almost unchanged over the incubation period of $6 \mathrm{~h}$ with only slight decrease from $8.4 \pm 0.03 \mathrm{~ns}$ (time 0$)$ to $8.2 \pm 0.06 \mathrm{~ns}(6 \mathrm{~h})$. On the other hand, treatment with bacterial collagenase significantly decreased the AFL in every time point over the $6 \mathrm{~h}$ period. The data suggest that decrease of AFL by the enzyme treatment is indeed a result of action of enzyme to the cartilage matrix.

\section{Effect of aggrecan degradation and interstitial collagenase, MMP-1}

We next investigated the effect of aggrecan removal and collagen removal in cartilage in selective manner to . As shown in Figure 5A, trypsin at $100 \mu \mathrm{g} / \mathrm{ml}$ effectively degrades and removes aggrecan from the cartilage but does not degrade type II collagen. Treating cartilage with MMP-1 at $4 \mu \mathrm{g} / \mathrm{ml}$ also resulted in aggrecan removal up to $40 \%$, with modest collagen degradation of $10 \%$, suggesting that MMP1 acts on aggrecan first and then collagen. Treating live cartilage with retinoic acid induces expression of aggrecanases and removes aggrecan but not collagen from the cartilage effectively. As shown in Figure 5B, after treating cartilage for 3 days with retinoic acid, aggrecan in the cartilage was almost completely removed, but there are 
no collagen degradation observed.

We examined the effect of aggrecan removal by trypsin on AFL in porcine cartilage. As shown in Figure 5C, trypsin treatment reduced the AFL in a statistically significant manner. We next examined the effect of MMP-1 treatment on porcine cartilage. As shown in Figure 5D, treating cartilage with MMP-1 caused significant decrease of the mean AFL in a dose dependent manner. At a concentration of $50 \mu \mathrm{g} / \mathrm{ml}$, the significant decrease of AFL corresponded to about $50 \%$ of collagen released to culture medium (data not shown). Finally, we examined effect of aggrecan removal by inducing expression of endogenous aggrecanses with retinoic acid (RA) treatment (Flannery et al., 1999). As shown in Figure 5E, cartilage treated with RA exhibited significantly lower AFL.

\section{Fluorescence lifetime imaging of cartilage tissue treated with collagenase}

To confirm the data obtained above using the single-point fibre-optic probe AFL measurements, we also utilized wide-field fluorescence lifetime microscopy. For this, a cartilage piece was split into halves and one piece was incubated in the buffer while the other piece was treated either with bacterial collagenase or human MMP-1 for 24 and 48 hours, respectively. For buffer control samples, one piece was incubated in the buffer and the other froze down immediately without incubation. After incubation, the two halves of cartilages were placed back together and the interface imaged with the fluorescence lifetime microscope. As shown in Figure 6A, incubating cartilage pieces in the buffer for 48 hours did not result in any change in their AFL. On the other hand, incubating with bacterial collagenase or MMP-1 caused a reduction of AFL compared to the cartilage pieces incubated in the buffer alone. Due to the relatively low fluorescence signal recorded in each pixel of the 
autofluorescence image, the decay data was fitted to a single exponential decay model, since there were insufficient photons to accurately fit a double exponential decay model. While this is less rigorous, yielding noticeable differences in AFL values $(\sim 6$ ns) compared to the double exponential fits to the data obtained with the single-point fluorometer probe (typically $\sim 8-10 \mathrm{~ns}$ ), it does permit the lifetime contrast between different regions of the sample to be visualised, clearly showing that the degraded area of cartilage showed significantly lower lifetimes relative to intact area of cartilage. These results confirm the trends observed using the multidimensional fluorometer, indicating that cartilage matrix degradation has led to a detectable decrease in AFL and suggesting that AFL may provide useful to identify degraded areas of cartilage.

\section{DISCUSSION}

The function of cartilage depends on its extracellular matrix (ECM). Collagen provides tissue architecture and tensile strength to the tissue, and aggrecan provides its compression resistant property by maintaining water molecules in the tissue. Thus, the degradation of these ECM components severely compromises the joint function. In this manuscript, we demonstrate that AFL can be a potential label-free means to detect changes in cartilage matrix in a non-invasive manner. The advantage of AFL measurement is that the detection of autofluorescence of endogenous ECM components does not involve administration of exogenous fluorescent labels and therefore may provide a means to probe live tissues.

Upon proteolytic actions in the cartilage, the mean AFL decreased over time and according to the level of degradation. Bacterial collagenase, which can dissolve cartilage tissue effectively, significantly decreased the mean AFL. In this treatment, it 
is likely that the bacterial collagenase degrades both collagen and aggrecan as this preparation of bacterial collagenase (Type 2) is known to have a contamination of a trypsin-like enzyme. Indeed treating cartilage with $100 \mu \mathrm{g} / \mathrm{ml}$ for $24 \mathrm{~h}$ released $80 \%$ of collagen as well as $80 \%$ of aggrecan from the cartilage tissue (data not shown). To more specifically examine the effect of loss of aggrecan, cartilage tissue was treated with trypsin. Since aggrecan provides a viscous environment between the collagen fibrils, its removal was expected to affect the cartilage AFL. In our experiments the complete aggrecan removal by trypsin had a statistically significant and reproducible (figure 5C) effect on AFL. A similar decrease was also detected when live cartilage was treated with $10 \mu \mathrm{M}$ retinoic acids for three days to induce endogenous aggrecanases which removed aggrecan almost completely from the cartilage tissue. Treating cartilage with active form of mammalian collagenase, MMP-1, also decreased the AFL in a more significant manner as shown in figure 5D. During the course of the MMP-1 treatment, aggrecan was released in an unexpectedly efficient manner, and this was followed by collagen degradation. We found $50 \mu \mathrm{g} / \mathrm{ml} \mathrm{MMP-1}$ treatment resulted in about $50 \%$ of collagen release and $60 \%$ of aggrecan release from the cartilage. We therefore attribute the significant shift in the mean AFL observed for a relatively high concentration of MMP-1 to the both collagen degradation and aggrecan degradation. In cartilage, collagen exists as fibrils. The MMP-1 cleavage site in monomeric collagen locates at the three quarter from the Nterminus (Visse and Nagase, 2003). Within a fibril, the cleavage site of collagen is located at close to the telopeptides of other collagen molecules where inter-molecule crosslinking occurs (Perumal et al., 2008). It is thus possible that cleavage of collagen by MMP-1 affects the local environment of crosslinks that are sources of autofluorescence in collagen molecules and hence affects the AFL. In osteoarthritic 
cartilage, collagen degradation was found to occur progressively from the surface of the tissue and around the chondrocytes (Hollander et al., 1995). It is not clear which collagenolytic MMPs are playing a role in this collagenolysis, but it is expected that collagenolytic action on cartilage tissue by any of the collagenolytic MMPs including MMP-1, MMP-2, MMP-8, MMP-13 and MMP-14 would change the AFL since they all cleave the Gly-Leu or Gly-Gln of the same region in the collagen II molecule (Knäuper et al., 1996; Mitchell et al., 1996; Ohuchi et al., 1997; Patterson et al., 2001). Therefore AFL could provide a useful means to monitor cartilage matrix degradation.

The multidimensional fluorometer could be considerably simplified, e.g. by dispensing with the spectral resolution, such that it could become portable and clinically deployable, for example by adapting the fibre optic probe for in vivo "needle biopsy". Furthermore, a FLIM instrument could be developed for arthroscopic application (Requejo-Isidro et al., 2004) and so provide a novel means to image the status of cartilage in a non-invasive manner. It is therefore important to further validate AFL measurements of cartilage degradation and this will be the subject of future investigations.

\section{EXPERIMENTAL PROCEDURES}

\section{Materials}

Bovine type-II collagen and trypsin was purchased from Sigma-Aldrich (Dorset, UK). Bacterial collagenase type 2 was purchased from Worthington Biochemical Corp (Lakewood, NJ, USA).

Recombinant MMP-1 was expressed in E.coli, purified and activated as previously reported (Chung et al., 2000; Jozic et al., 2005).

\section{Single-point fibre-optic multidimensional fluorometer probe}


The AFL measurements were undertaken using a single-point

multidimensional fluorometer implemented with a fibre-optic probe as described previously (Manning et al., 2008a). This instrument facilitates measurements of fluorescence resolved with respect to excitation and emission wavelength, fluorescence lifetime and polarisation. It was configured with a diode-pumped frequency-tripled Neodymium Vanadate laser (Vanguard 350-HM355, SpectraPhysics, USA) as the excitation source, which provides 12 ps pulses at $80 \mathrm{MHz}$ repetition rate centred on $355 \mathrm{~nm}$ wavelength. The custom-built fibre-optic probe (FiberTech Optica, Canada) incorporated three excitation fibres and 16 collection fibres. The fluorescence detection was a motorised monochromator (CM110, CVI Inc., USA) and a cooled photomultiplier (PMC-100, Becker-Hickl GmbH, Germany) to provide single channel spectrally resolved detection with time-resolution implemented using time-correlated single photon counting (SPC 730, Becker-Hickl $\mathrm{GmbH}$, Germany). The fitting of fluorescence lifetime data was undertaken using the software package TRFA (SSTC, Belarus).

\section{Cartilage explant}

Porcine articular cartilage and bovine nasal cartilage were obtained from slaughterhouse and were prepared as previously described (Gendron et al., 2003; Miller et al., 2009). Cartilage pieces (approx. $5 \mathrm{~mm}$ x $5 \mathrm{~mm}$ x $2.5 \mathrm{~mm}$ ) were frozen and thawed 3 times to kill chondrocytes and kept in $-20^{\circ} \mathrm{C}$ until they are subjected to experiments.

\section{Analysis of glycosaminoglycan (GAG) and hydroxyproline release}

GAG released into the conditioned medium was measured in triplicate using a modification of the DMMB assay as described previously (Farndale et al., 1986; Gendron et al., 2003). Shark chondroitin sulfate $(0-2.62 \mu \mathrm{g})$ was used as the standard. 
The percentage of total GAG released into the medium was calculated as follows: \% of total GAG released=(total GAG in the medium $) /($ total GAG in the medium+total GAG remaining in the cartilage). All data were analyzed by unpaired one-tail t tests with Welch's correction using the software package GraphPad Prism (San Diego, CA, USA). Hydroxyproline release into the conditioned medium was measured according to Woessner (Woessner, 1961). The amount released was calculated according to the standard curve. All data were analyzed by unpaired one-tail t tests with Welch's correction using the software package GraphPad Prism.

\section{Wide-field fluorescence lifetime imaging}

Fluorescence lifetime imaging was implemented in a wide-field microscope as described previously (Dowling et al., 1998; Talbot et al., 2010). Briefly, wide-field time-gated detection was implemented on a conventional wide-field inverted fluorescence microscope frame (Olympus IX81) configured for excitation at $365 \mathrm{~nm}$ using an 80MHz frequency-doubled Ti:Sapphire laser (MaiTai, Spectra Physics). Wide-field FLIM was implemented using a gated optical image intensifier (HRI, Kentech Instruments Ltd, Didcot, UK) read out by a cooled CCD camera (Orca-ER, Hamamatsu Photonics KK, Shizuoka-Ken, Japan). 


\section{Acknowledgments}

This work was funded by the Biotechnology and Biological Sciences Research

Council (BBSRC BB/E000495/1) and the Engineering and Physical Sciences

Research Council (EPSRC EP/F040202/1 and EPSRC EP/IO2770X/1) and the

Arthritis Research UK core grant to the Kennedy Institute. Hugh Manning was funded

by a studentship from the EPSRC-funded Chemical Biology Centre Doctoral Training

Centre. Paul French acknowledges a Royal Society Wolfson Research Merit Award. 


\section{References}

Chung, L., Shimokawa, K., Dinakarpandian, D., Grams, F., Fields, G.B., Nagase, H., 2000. Identification of the (183)RWTNNFREY(191) region as a critical segment of matrix metalloproteinase 1 for the expression of collagenolytic activity. J Biol Chem 275, 29610-29617.

Dowling, K., Dayel, M.J., Lever, M.J., French, P.M., Hares, J.D., Dymoke-Bradshaw, A.K., 1998. Fluorescence lifetime imaging with picosecond resolution for biomedical applications. Optics letters 23, 810-812.

Elson, D., Requejo-Isidro, J., Munro, I., Reavell, F., Siegel, J., Suhling, K., Tadrous, P., Benninger, R., Lanigan, P., McGinty, J., Talbot, C., Treanor, B., Webb, S., Sandison, A., Wallace, A., Davis, D., Lever, J., Neil, M., Phillips, D., Stamp, G., French, P., 2004. Time-domain fluorescence lifetime imaging applied to biological tissue. Photochem Photobiol Sci 3, 795-801.

Farndale, R.W., Buttle, D.J., Barrett, A.J., 1986. Improved quantitation and discrimination of sulphated glycosaminoglycans by use of dimethylmethylene blue. Biochim Biophys Acta 883, 173-177.

Flannery, C.R., Little, C.B., Hughes, C.E., Caterson, B., 1999. Expression of ADAMTS homologues in articular cartilage. Biochem Biophys Res Commun 260, 318-322.

Gendron, C., Kashiwagi, M., Hughes, C., Caterson, B., Nagase, H., 2003. TIMP-3 inhibits aggrecanase-mediated glycosaminoglycan release from cartilage explants stimulated by catabolic factors. FEBS Lett 555, 431-436.

Georgakoudi, I., Jacobson, B.C., Müller, M.G., Sheets, E.E., Badizadegan, K., CarrLocke, D.L., Crum, C.P., Boone, C.W., Dasari, R.R., Van Dam, J., Feld, M.S., 2002. $\mathrm{NAD}(\mathrm{P}) \mathrm{H}$ and collagen as in vivo quantitative fluorescent biomarkers of epithelial precancerous changes. Cancer Res 62, 682-687.

Glasson, S.S., Askew, R., Sheppard, B., Carito, B., Blanchet, T., Ma, H.L., Flannery, C.R., Peluso, D., Kanki, K., Yang, Z., Majumdar, M.K., Morris, E.A., 2005. Deletion of active ADAMTS5 prevents cartilage degradation in a murine model of osteoarthritis. Nature 434, 644-648.

Heinegård, D., Lorenzo, P., Saxne, T., 2001. Matrix Glycoproteins, Proteoglycans, and Cartilage, in: S, R., Harris, E.J., CB, S. (Eds.), Kelly's Textbook of Rheumatology, 6 ed. W.B. Saunders Company, Philadelphia, pp. 41-53

Hollander, A.P., Pidoux, I., Reiner, A., Rorabeck, C., Bourne, R., Poole, A.R., 1995. Damage to type II collagen in aging and osteoarthritis starts at the articular surface, 
originates around chondrocytes, and extends into the cartilage with progressive degeneration. J Clin Invest 96, 2859-2869.

Jozic, D., Bourenkov, G., Lim, N.H., Visse, R., Nagase, H., Bode, W., Maskos, K., 2005. X-ray structure of human proMMP-1: new insights into procollagenase activation and collagen binding. J Biol Chem 280, 9578-9585.

Knäuper, V., Lopez-Otin, C., Smith, B., Knight, G., Murphy, G., 1996. Biochemical characterization of human collagenase-3. J Biol Chem 271, 1544-1550.

Lakowicz, J.R., 2006. Principles of Fluorescence Spectroscopy, Third Edition, 2 ed. Springer.

Manning, H.B., Kennedy, G.T., Owen, D.M., Grant, D.M., Magee, A.I., Neil, M.A., Itoh, Y., Dunsby, C., French, P.M., 2008a. A compact, multidimensional spectrofluorometer exploiting supercontinuum generation. J Biophotonics 1, 494-505.

Manning, H.B., Kennedy, G.T., Owen, D.M., Grant, D.M., Magee, A.I., Neil, M.A.A., Itoh, Y., Dunsby, C., French, P.M.W., 2008b. A compact, multidimensional spectrofluorometer exploiting supercontinuum generation. J Biophotonics 1, 494-505.

Menter, J.M., 2006. Temperature dependence of collagen fluorescence. Photochem. Photobiol. Sci. 5, 403-410.

Miller, M.C., Manning, H.B., Jain, A., Troeberg, L., Dudhia, J., Essex, D., Sandison, A., Seiki, M., Nanchahal, J., Nagase, H., Itoh, Y., 2009. Membrane type 1 matrix metalloproteinase is a crucial promoter of synovial invasion in human rheumatoid arthritis. Arthritis Rheum 60, 686-697.

Mitchell, P.G., Magna, H.A., Reeves, L.M., Lopresti-Morrow, L.L., Yocum, S.A., Rosner, P.J., Geoghegan, K.F., Hambor, J.E., 1996. Cloning, expression, and type II collagenolytic activity of matrix metalloproteinase-13 from human osteoarthritic cartilage. J Clin Invest 97, 761-768.

Murphy, G., Nagase, H., 2008. Reappraising metalloproteinases in rheumatoid arthritis and osteoarthritis: destruction or repair? Nat Clin Pract Rheumatol 4, 128-135.

Ohuchi, E., Imai, K., Fujii, Y., Sato, H., Seiki, M., Okada, Y., 1997. Membrane type 1 matrix metalloproteinase digests interstitial collagens and other extracellular matrix macromolecules. J Biol Chem 272, 2446-2451.

Patterson, M.L., Atkinson, S.J., Knauper, V., Murphy, G., 2001. Specific collagenolysis by gelatinase A, MMP-2, is determined by the hemopexin domain and not the fibronectin-like domain. FEBS Lett 503, 158-162. 
Perumal, S., Antipova, O., Orgel, J.P., 2008. Collagen fibril architecture, domain organization, and triple-helical conformation govern its proteolysis. Proc Natl Acad Sci U S A 105, 2824-2829.

Requejo-Isidro, J., McGinty, J., Munro, I., Elson, D.S., Galletly, N.P., Lever, M.J., Neil, M.A., Stamp, G.W., French, P.M., Kellett, P.A., Hares, J.D., Dymoke-Bradshaw, A.K., 2004. High-speed wide-field time-gated endoscopic fluorescence-lifetime imaging. Optics letters 29, 2249-2251.

Sledge, C., Reddi, A., Walsh, D., Blake, D., 2001. Biology of the Normal Joint, in: S, R., Harris, E.J., CB, S. (Eds.), Kelly's Textbook of Rheumatology, 6 ed. W.B. Saunders Company, Philadelphia, pp. 1-26.

Stanton, H., Melrose, J., Little, C.B., Fosang, A.J., 2011. Proteoglycan degradation by the ADAMTS family of proteinases. Biochim Biophys Acta 1812, 1616-1629.

Stanton, H., Rogerson, F.M., East, C.J., Golub, S.B., Lawlor, K.E., Meeker, C.T., Little, C.B., Last, K., Farmer, P.J., Campbell, I.K., Fourie, A.M., Fosang, A.J., 2005. ADAMTS5 is the major aggrecanase in mouse cartilage in vivo and in vitro. Nature $434,648-652$.

Talbot, C., McGinty, J., McGhee, E., Owen, D.M., Grant, D.M., Kumar, S., De Beule, P., Auksorius, E., Manning, H.B., Galletly, G., Treanor, B., Kennedy, G.T., Lanigan, P.M.P., Munro, I., Elson, D.S., McGee, A., Davis, D., Neil, M., Stamp, G., Dunsby, C., French, P., 2010. Fluorescence lifetime imaging and metrology for biomedicine, in: Tuchin, V. (Ed.). CRC Press, Taylor and Francis Group, pp. 159-196.

Visse, R., Nagase, H., 2003. Matrix metalloproteinases and tissue inhibitors of metalloproteinases: structure, function, and biochemistry. Circ Res 92, 827-839.

Wagnieres, G.A., Star, W.M., Wilson, B.C., 1998. In vivo fluorescence spectroscopy and imaging for oncological applications. Photochem Photobiol 68, 603-632.

Woessner, J.F., Jr., 1961. The determination of hydroxyproline in tissue and protein samples containing small proportions of this imino acid. Archives of biochemistry and biophysics 93, 440-447.

Wu, W., Billinghurst, R.C., Pidoux, I., Antoniou, J., Zukor, D., Tanzer, M., Poole, A.R., 2002. Sites of collagenase cleavage and denaturation of type II collagen in aging and osteoarthritic articular cartilage and their relationship to the distribution of matrix metalloproteinase 1 and matrix metalloproteinase 13. Arthritis Rheum 46, 2087-2094. 


\section{FIGURE LEGENDS}

\section{Figure 1. Excitation-emission matrix of bovine type II collagen}

Excitation-emission matrix of lyophilized type II collagen recorded using a commercial spectrofluorometer (Shimadzu). Pseudocolor scale represents relative fluorescence intensity in arbitrary units where the highest is red and lowest is dark blue. Reported sources of autofluorescence in collagen are indicated with arrows (Lakowicz, 2006).

\section{Figure 2. Auto-fluorescence lifetime of cartilage tissue}

(A) Time-resolved emission spectrum and (B) time-integrated emission spectrum of fresh porcine articular cartilage excited at $355 \mathrm{~nm}$ by 12 ps pulses at $80 \mathrm{MHz}$ repetition rate measured using multidimensional fluorometer via fibre optic probe. (C) Cartilage autofluorescence decay profile (blue curve) acquired at $355 \mathrm{~nm} / 415 \mathrm{~nm}$ excitation/emission with instrument response function (IRF, black curve) and double exponential fit (red curve) with residuals to fit (green curve). (D) Dot plot of mean AFL measurements of frozen and thawed porcine articular cartilage $(n=21)$. The AFL value is $9.9 \pm 0.2 \mathrm{~ns}$ (mean $\pm \mathrm{SEM})$.

\section{Figure 3. Effect of collagnese on the AFL of porcine articular cartilage}

(A) Fluorescence decay profiles of porcine articular cartilage treated with different concentration of bacterial collagenase ( 0 to $100 \mu \mathrm{g} / \mathrm{ml}$ ) at $37^{\circ} \mathrm{C}$ for $16 \mathrm{~h}$ as indicated. (B) Mean AFL of porcine articular cartilage treated with different concentrations of bacterial collagenase. Error bars (SEM) shown are from five separate measurements each on five different cartilages of each dose. A linear fit to the data point is shown with $A F L=39.25-0.04[C]$, where $[C]$ is the concentration of collagenase $\left(r^{2}=0.90\right.$. 
(C) Percentage of autofluorescence photons emitted from the shorter lifetime component, showing that the contribution of the shorter component increases in a dose dependent manner. The lifetime of the longer component decreased with concentration (data not shown).

(D) Time-integrated and normalised fluorescence emission spectra excited at $355 \mathrm{~nm}$ of cartilage treated with $100 \mu \mathrm{g} / \mathrm{ml}$ collagenase for $16 \mathrm{~h}$ at $37^{\circ} \mathrm{C}$ (red) or with the buffer control (blue).

Figure 4. Time-dependent effect of bacterial collagenase-treatment on Cartilage AFL

Cartilage samples were treated with $(n=3)$ or without $(n=3)$ bacterial collagenase $(200$ $\mu \mathrm{g} / \mathrm{ml}$ ) at $37{ }^{\circ} \mathrm{C}$ over the period of $6 \mathrm{~h}$. AFL of the same area of the same cartilage piece was measured at each time point, and plotted. $* P<0.013 ; * * P<0.007$; $* * * P<0.0004$

Figure 5. Effect of trypsin or MMP-1 treatment on cartilage AFL

(A) Freeze and thawed porcine articular cartilage treated with trypsin $(2.5 \mu \mathrm{g} / \mathrm{ml})$ or MMP-1 $(4 \mu \mathrm{g} / \mathrm{ml})$ or in the buffer alone at $37^{\circ} \mathrm{C}$ for $72 \mathrm{~h}$. Supernatants were collected and subjected to DMMB assay for aggrecan release and Hydroxy proline (Hy-Pro) assay for collagen release $(n=12)$.

(B) Live porcine articular cartilage was treated with or without $10 \mu \mathrm{M}$ retinoic acid (RA) for three days. Culture supernatants were collected and subjected to DMMB assay for aggrecan release and Hy-Pro assay for collagen release $(n=10)$.

(C) Mean AFL of porcine cartilage samples treated with or without trypsin (100 $\mu \mathrm{g} / \mathrm{ml}$ ) at $37^{\circ} \mathrm{C}$ for $24 \mathrm{~h}$ and measured using multidimensional fluorometer via fiber- 
optic probe $(\mathrm{n}=9$ for control and 8 for trypsin. $* * * p<0.0009)$.

(D) Mean AFL of porcine cartilage treated with MMP-1 $(50 \mu \mathrm{g} / \mathrm{ml})$ at $37{ }^{\circ} \mathrm{C}$ for $96 \mathrm{~h}$. Mean AFL are indicated with SEM. $(\mathrm{n}=5) * * * p<0.0009$, ****p $<<0.0002$, $* * * * * p<0.0001$

(E) Mean AFL of porcine cartilage treated with RA. Mean AFL are indicated with SEM.

\section{Figure 6. FLIM cartilage degradation by collagenase}

(A) Autofluorescence lifetime images fitted to a single exponential decay model

(AFLIM, upper row) and time-integrated intensity images (lower row) of autofluorescence of cartilage pieces. A cartilage piece was halved and one piece was soaked in the buffer containing with or without bacterial collagenase $(50 \mu \mathrm{g} / \mathrm{ml})$ for $24 \mathrm{~h}$ or human active MMP-1 $(50 \mu \mathrm{g} / \mathrm{ml})$ for $48 \mathrm{~h}$ in humidified incubator at $37{ }^{\circ} \mathrm{C}$. The other piece of cartilage was either frozen down immediately (Buffer control) or incubated with buffer without enzymes for the same length of time as the other piece. Two paired pieces of cartilages were then put together, and interface of cartilages were imaged using the wide-field FLIM microscope. AFLIM maps have been merged with the intensity data and shown. (B) Bar charts showing mean lifetime differences between the control and treated cartilage for the different conditions. 


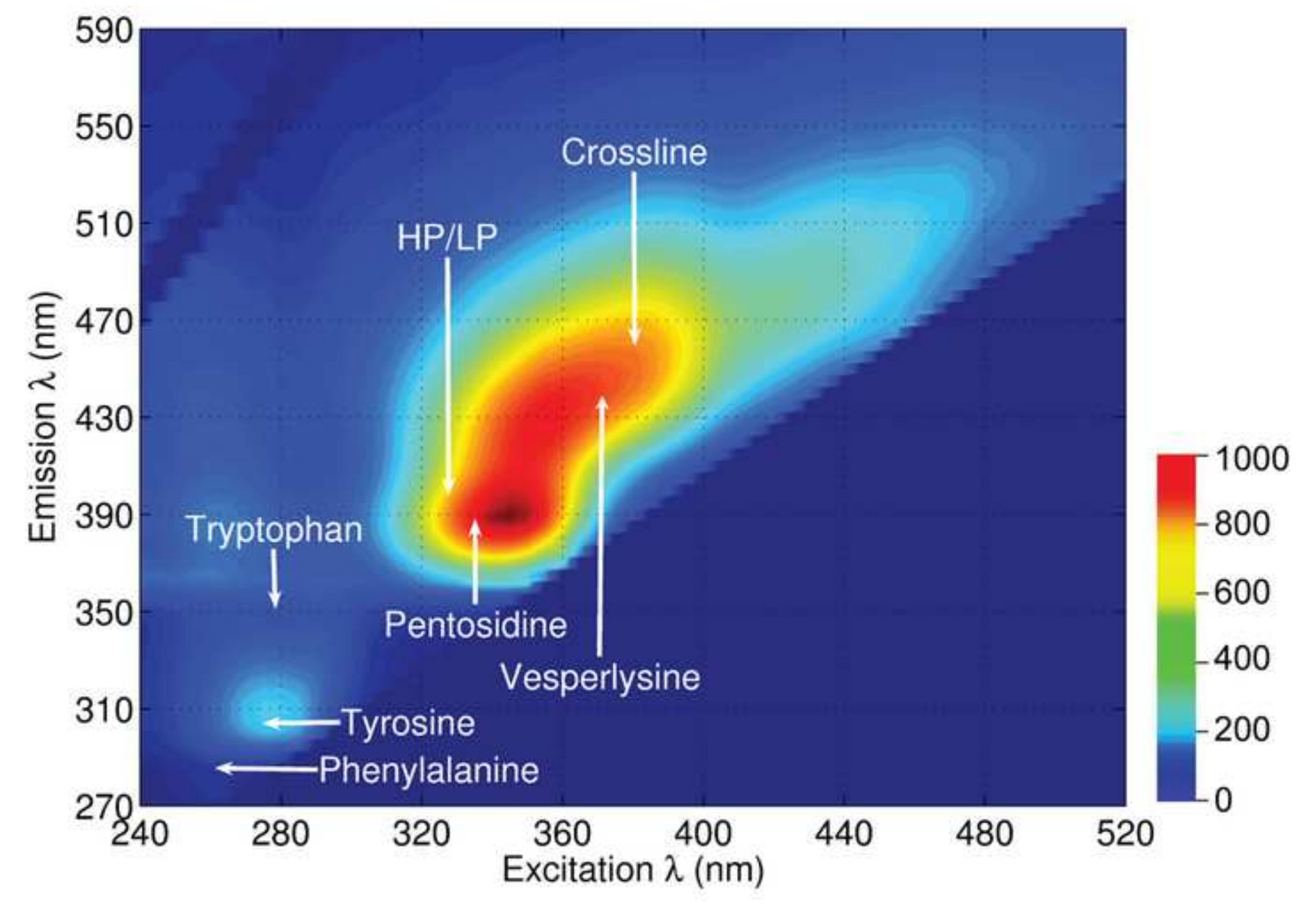


A

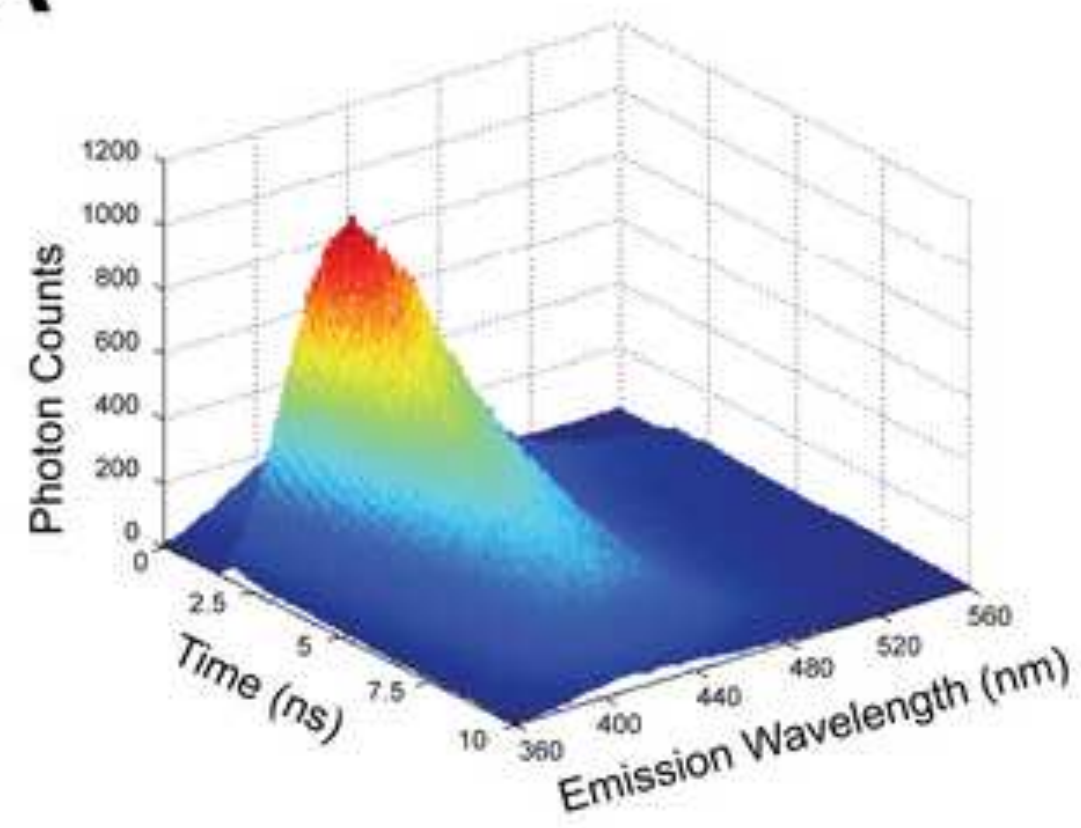

B

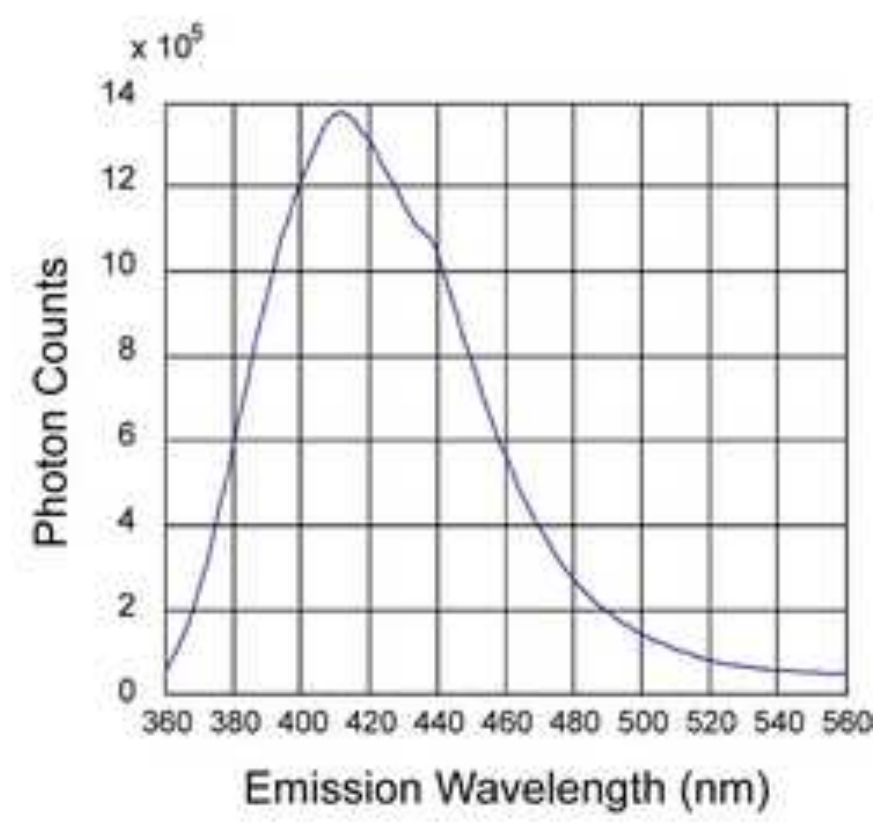

C

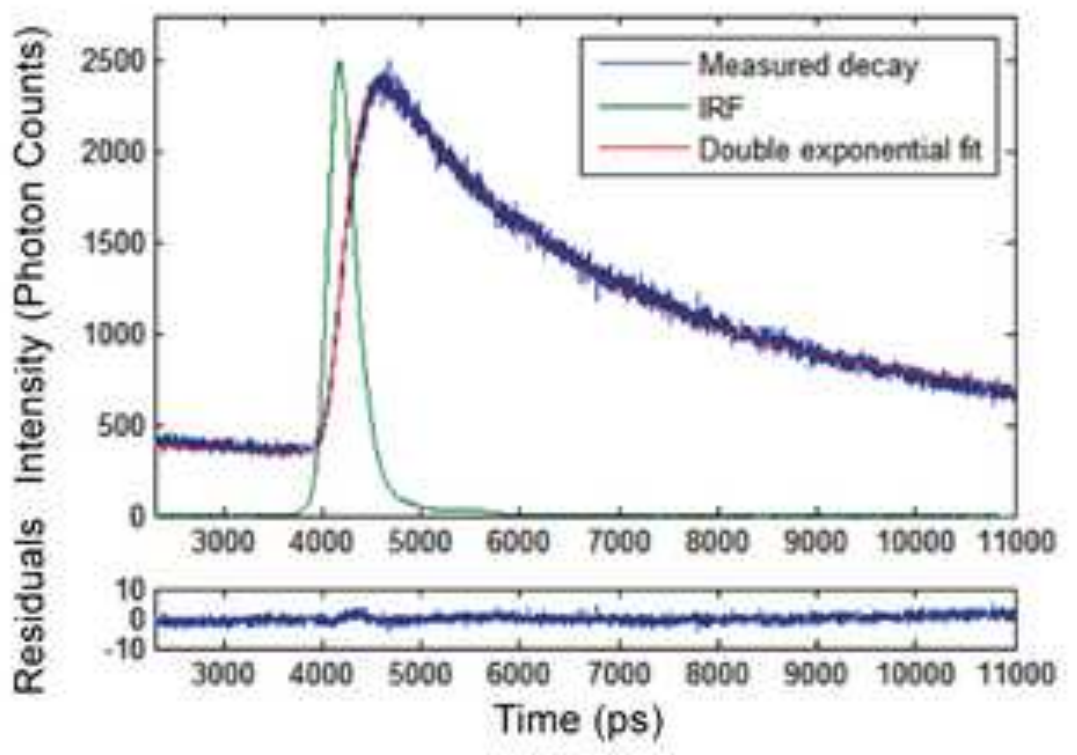

D

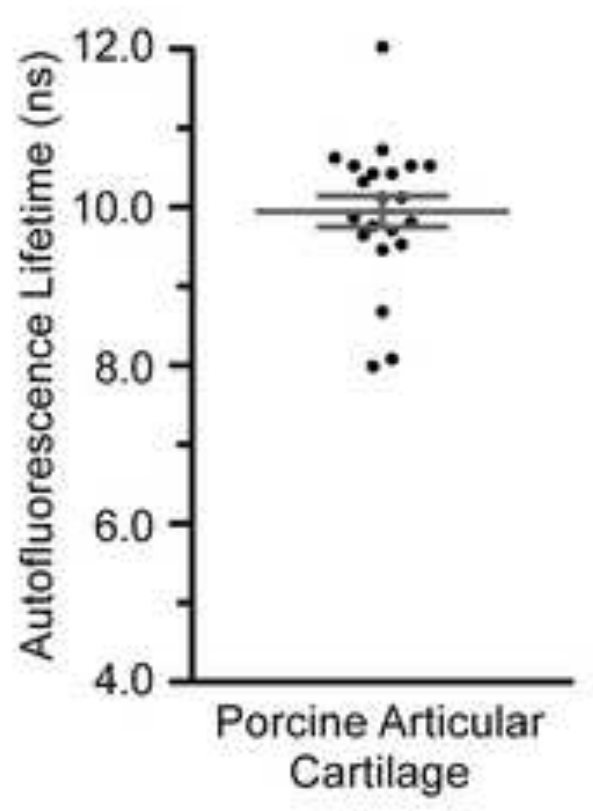


A

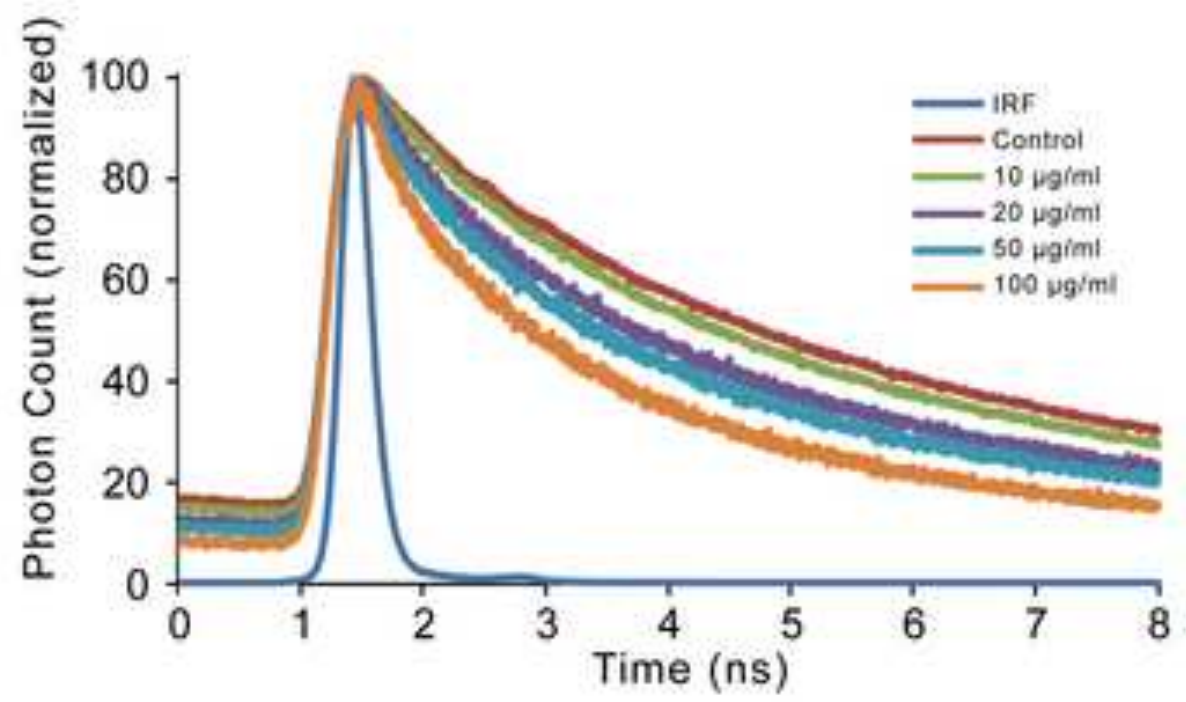

C

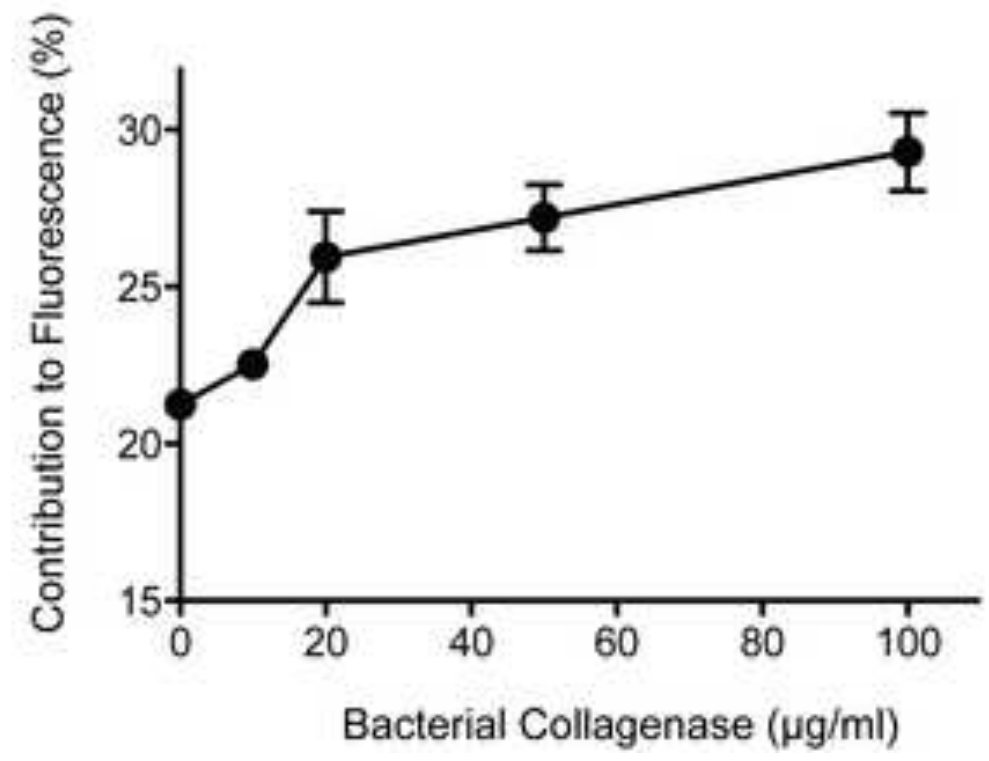

B

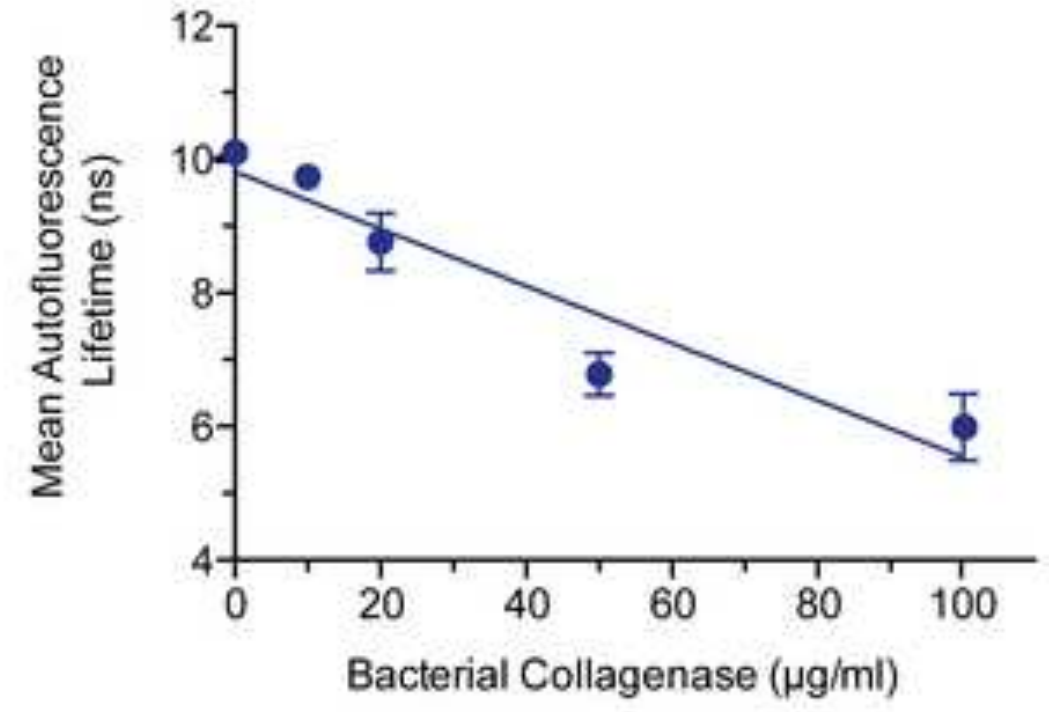

D

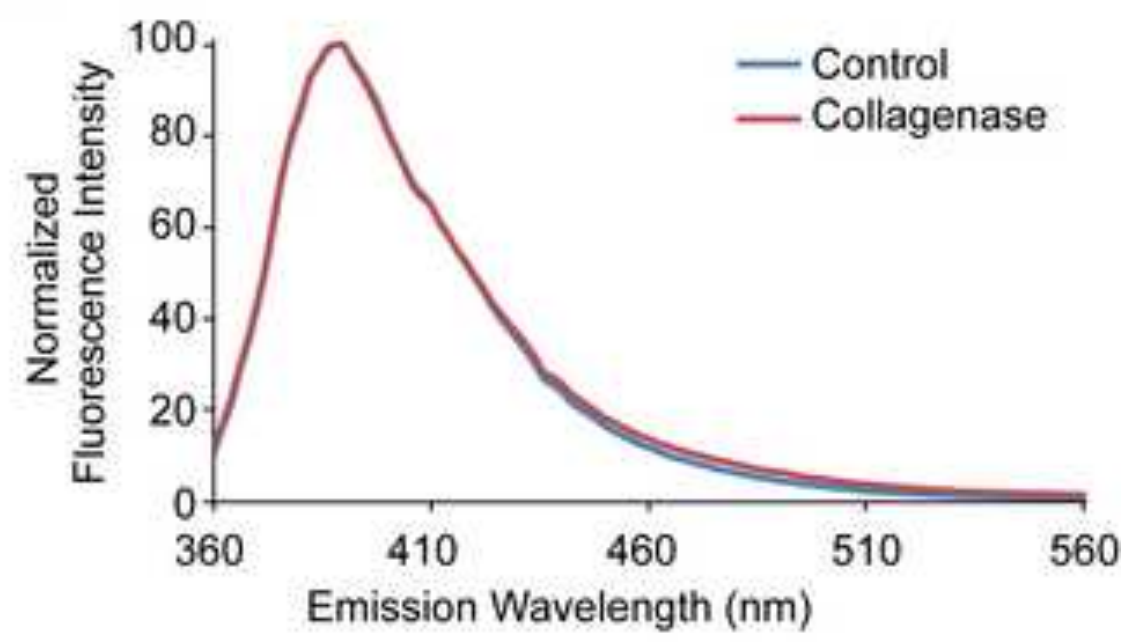




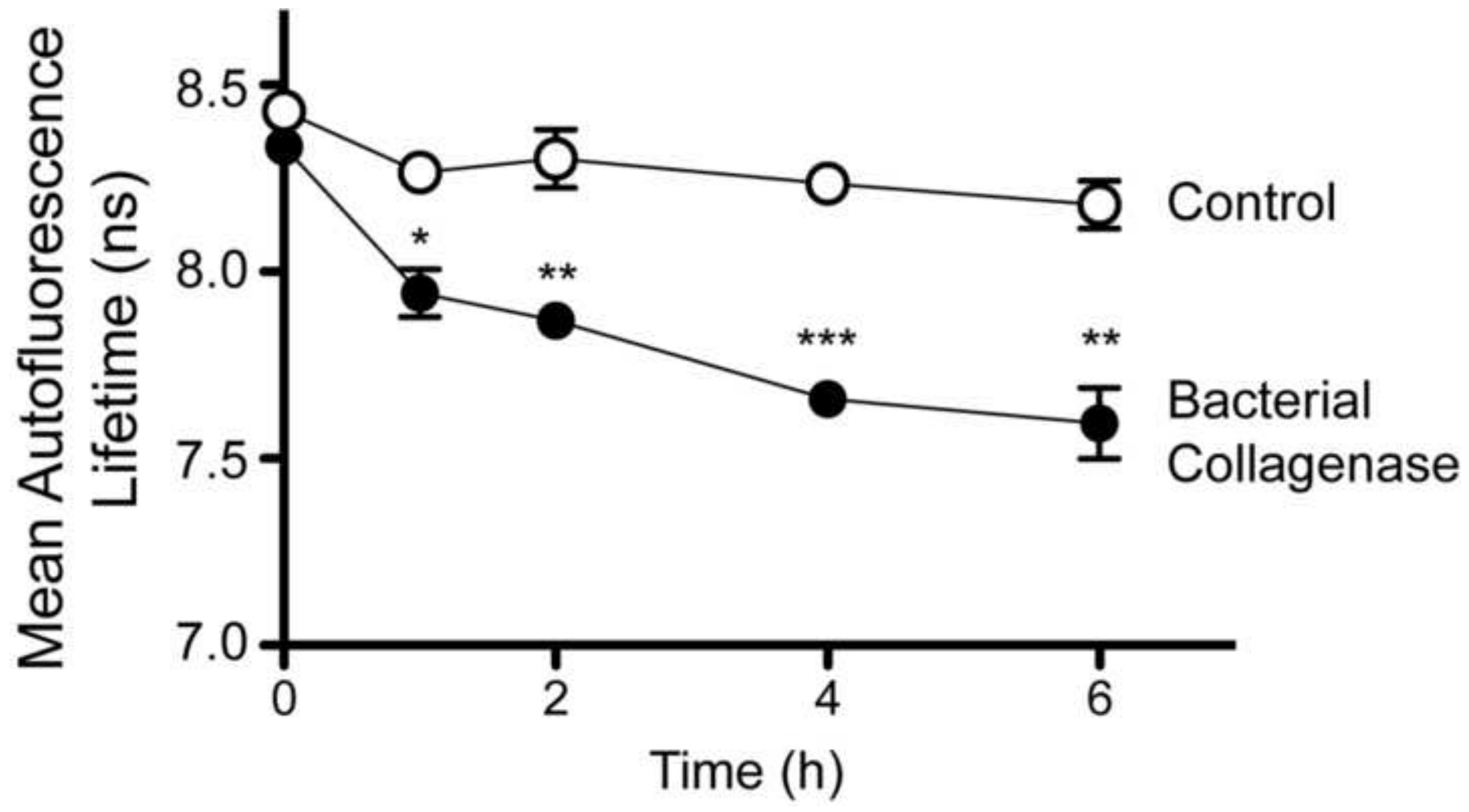


A

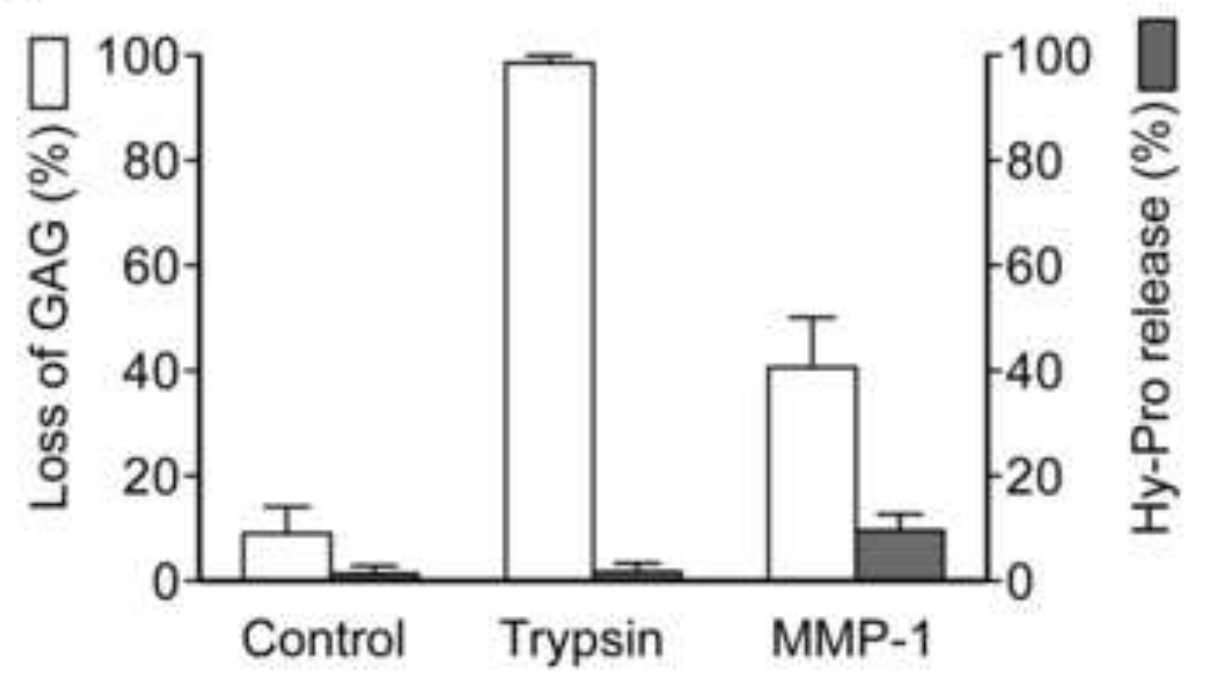

C

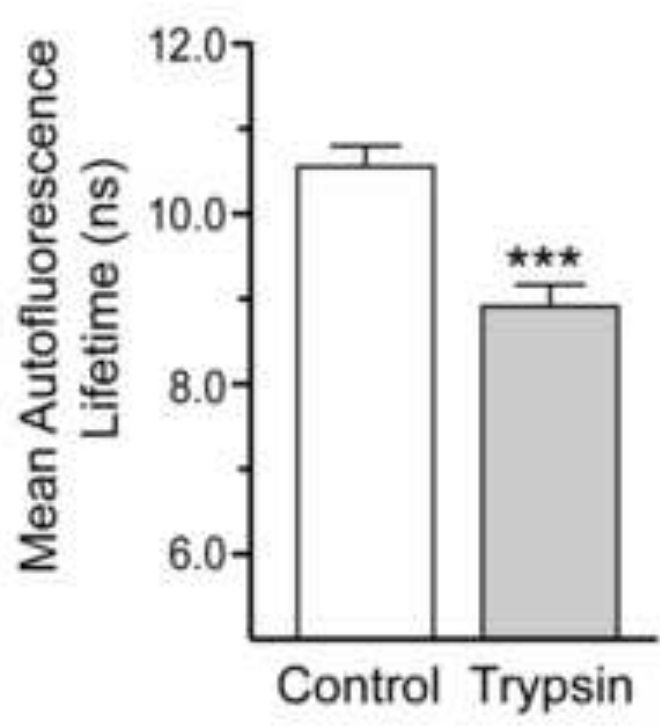

B

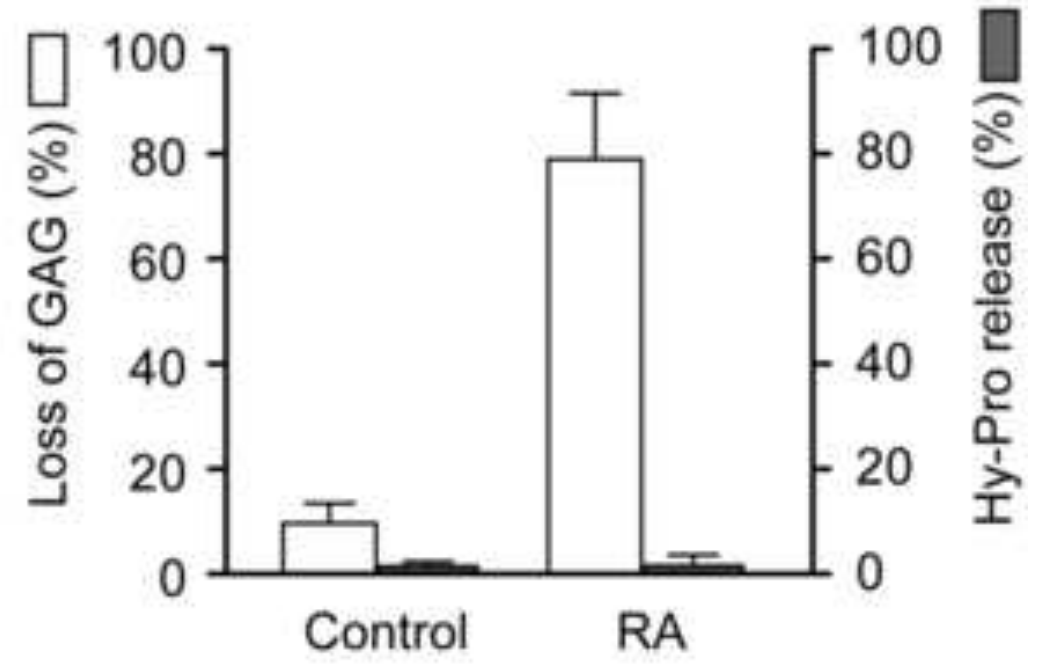

E

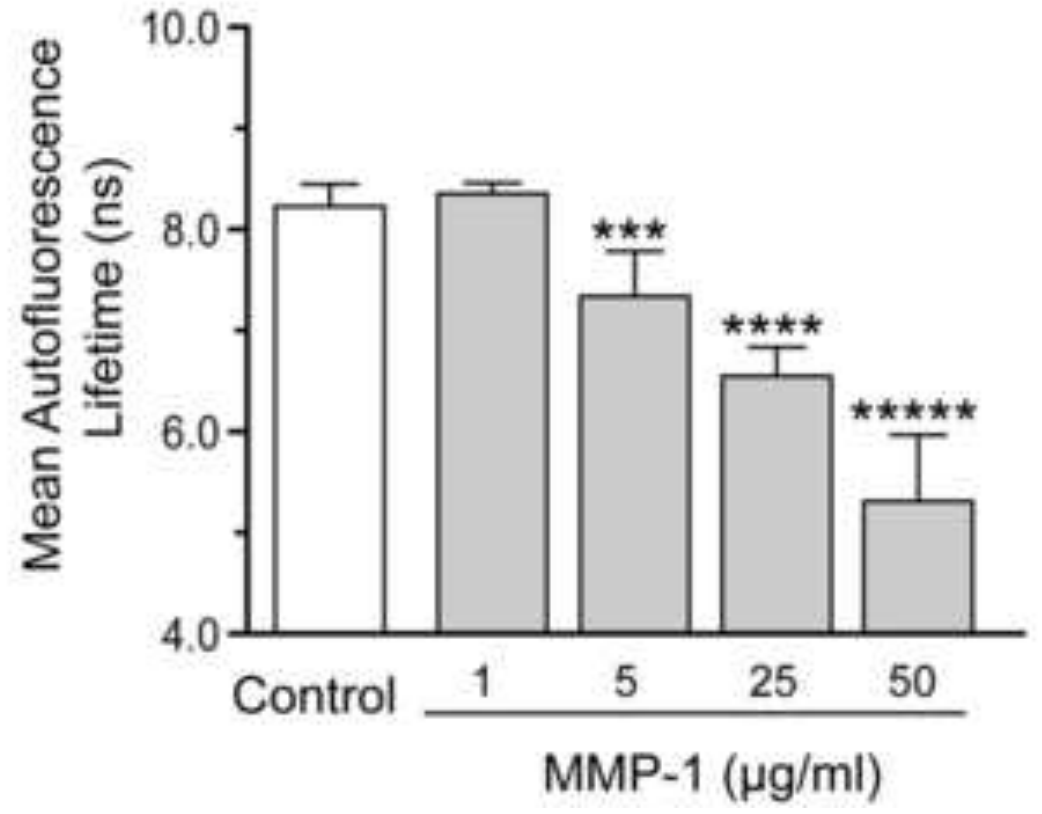

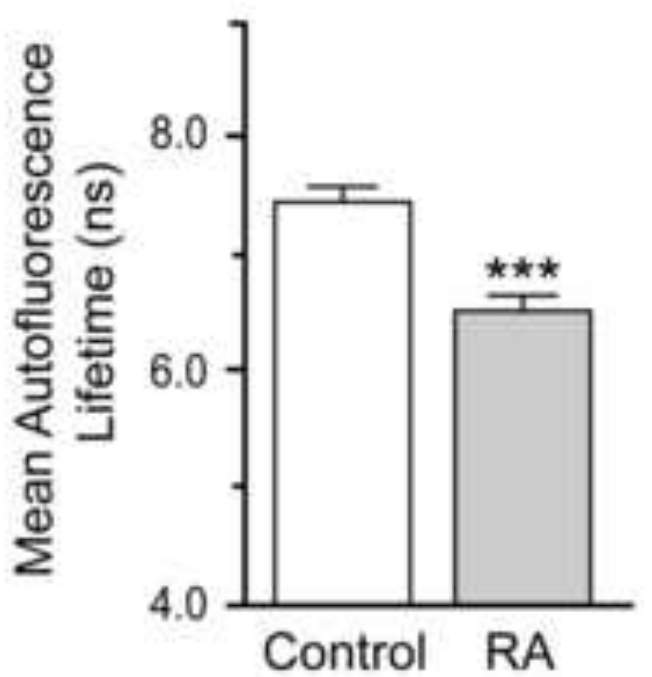


Click here to download high resolution image
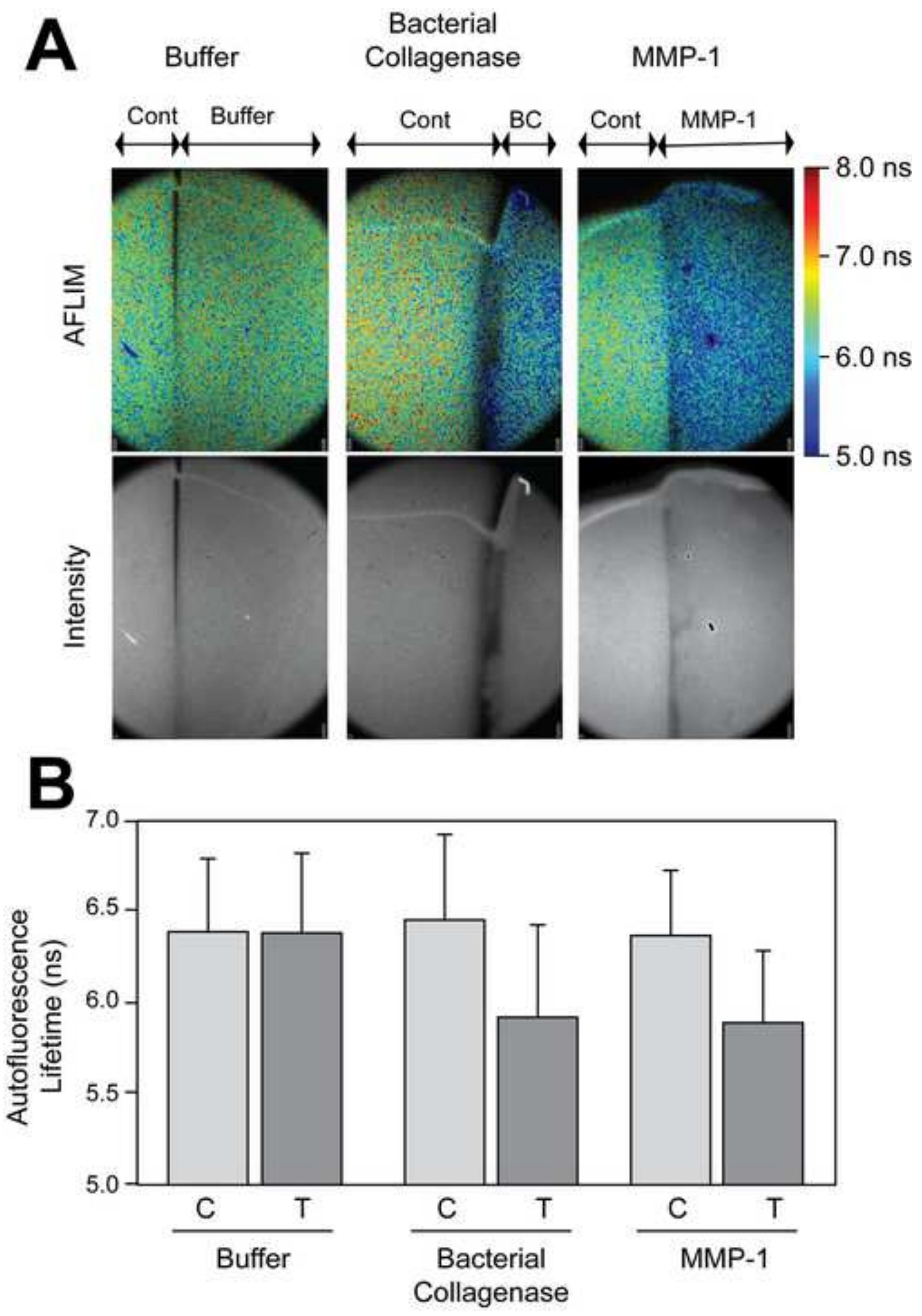